\title{
Strategic control over saccadic eye movements: Studies of the fixation offset effect
}

\author{
LIANA MACHADO and ROBERT D. RAFAL \\ University of Wales, Bangor, Wales
}

\begin{abstract}
We studied the strategic (presumably cortical) control of ocular fixation in experiments that measured the fixation offset effect (FOE) while manipulating readiness to make reflexive or voluntary eye movements. The visual grasp reflex, which generates reflexive saccades to peripheral visual signals, reflects an opponent process in the superior colliculus (SC) between fixation cells at the rostral pole, whose activity helps maintain ocular position and increases when a stimulus is present at fixation, and movement cells, which generate saccades and are inhibited by rostral fixation neurons. Voluntary eye movements are controlled by movement and fixation cells in the frontal eye field (FEF). The FOE-a decrease in saccade latency when the fixation stimulus is extinguished-has been shown to reflect activity in the collicular eye movement circuitry and also to have an activity correlate in the FEF. Our manipulation of preparatory set to make reflexive or voluntary eye movements showed that when reflexive saccades were frequent and voluntary saccades were infrequent, the FOE was attenuated only for reflexive saccades. When voluntary saccades were frequent and reflexive saccades were infrequent, the FOE was attenuated only for voluntary saccades. We conclude that cortical processes related to task strategy are able to decrease fixation neuron activity even in the presence of a fixation stimulus, resulting in a smaller FOE. The dissociation in the effects of a fixation stimulus on reflexive and voluntary saccade latencies under the same strategic set suggests that the FOEs for these two types of eye movements may reflect a change in cellular activity in different neural structures, perhaps in the SC for reflexive saccades and in the FEF for voluntary saccades.
\end{abstract}

The function of the oculomotor system in humans, as in all foveate animals, is to align objects of potential interest on retinal regions of high acuity and to maintain this alignment for continued processing even if the observer or the object moves. This is accomplished through opponent neural processes that move the eyes toward new objects and that maintain fixation on an object of current interest (Büttner-Ennever \& Horn, 1997). Here, we consider control over reflexive and voluntary eye movements and, especially, the mechanisms responsible for strategic control of fixation.

The visual grasp reflex (VGR) is a rapid eye movement toward a visual stimulus appearing in the periphery. This reflex is highly adaptive for survival because, for example, it enables us to quickly foveate an approaching stimulus, maximizing the amount of time we have to identify it and to respond. The cells that mediate the VGR are located in the superior colliculi (SCs). In the rostral pole of each SC are cells that are active during fixation (even in the dark) and whose activity is further increased by a vi-

This research was supported by PHS Grants RO1 MH41544 and RO1 MH51400 to R.D.R. Special thanks are due Tony Ro for programming assistance, Ilya Bushev for assisting with data collection, and John Henderson, Raymond Klein, Patti Reuter-Lorenz, and one anonymous reviewer for very helpful comments on this manuscript. Correspondence concerning this article should be addressed to L. Machado, Psychology Department, University of Wales, Bangor LL57 2DG, Wales (e-mail: 1.machado@bangor.ac.uk). sual signal at fixation. These fixation, or pole, neurons help keep the eyes from moving. Caudal to the fixation neurons, and inhibited by them, are cells - commonly referred to as movement cells 1 - whose activity helps the eyes move to a new position. Together, the activity of these two types of cells helps determine when and where your eyes will move (reviewed in Wurtz \& Munoz, 1995).

The offset of a fixated stimulus prior to, or simultaneous with, the onset of a peripheral target disinhibits the VGR and speeds reaction time to initiate an eye movement to the target. The benefit of fixation offset on saccadic latencies was first reported by Saslow (1967) and has been termed the fixation offset effect (FOE; Klein \& Kingstone, 1993). Fixation offset paradigms compare latencies to make eye movements when a fixation stimulus remains present at target onset with when the fixation stimulus offsets when the target onsets. Whether a fixation stimulus remains present when a target onsets is also manipulated in gap paradigms, but on fixation offset trials, a temporal gap is introduced between fixation offset and target onset. A temporal gap of $200 \mathrm{msec}$ produces the shortest saccadic latencies; sometimes, a separate population of very short latency saccades, termed express saccades, emerges (Fischer \& Ramsperger, 1984). The reduction in saccade latency caused by removing a fixated stimulus prior to target onset (the gap effect) reflects the FOE, owing to the absence of a fixation stimulus, plus a general alerting effect, afforded by the temporal gap (Kingstone \& Klein, 1993a). Thus, although the FOE is specific 
to the oculomotor system (Reuter-Lorenz, Hughes, \& Fendrich, 1991), the gap effect benefits other systems as well (Ross \& Ross, 1981). Moreover, the oculomotorspecific FOE requires removal of a stimulus at fixation, whereas the gap effect occurs for both central and peripheral offsets (Fendrich, Demirel, \& Danziger, 1999).

Fixation offset paradigms can be used to study the VGR as an opponent process. The FOE is thought to result from changes in cellular activity in local inhibitory circuits within the SCs that are triggered by the offset of a fixated stimulus (Munoz \& Istvan, 1998). When a fixated stimulus offsets, the activity of fixation neurons decreases, and eye movement latency is reduced (Dorris \& Munoz, 1995; Munoz \& Wurtz, 1992). Conversely, stimulating fixation neurons just prior to or during an eye movement can delay or arrest the eye movement (for the monkey, see Munoz \& Wurtz, 1993a; for the cat, see Paré \& Guitton, 1994). This finding is consistent with the demonstration that unilateral microstimulation of the rostral SC inhibits movement cells in both SCs (Munoz \& Istvan, 1998; Munoz \& Wurtz, 1993b). Together, these neurophysiological findings suggest that when fixation cell activity decreases in response to fixation offset, movement cell activity increases, disinhibiting the VGR and speeding reaction time to initiate an eye movement to a peripheral target.

The fixation reflex, in which a visual signal at the point of fixation reflexively activates fixation neurons that inhibit the VGR to eccentric events, is especially strong in early infancy (Johnson, 1990). At about 2 months of age, the colliculi come under the unopposed inhibitory influence of the basal ganglia (substantia nigra pars reticulata). Infants may become distressed because they are unable to break the lock of a visual stimulus in order to move their eyes. The FOE decreases during infant development (Hood, Atkinson, \& Braddick, 1998; Johnson \& Gilmore, 1998), marking a maturation of fronto-basal ganglia-collicular circuits that brings the fixation reflex under voluntary control, permitting efficient visual search with alternating saccades and fixations.

Moreover, normal adults can learn to make express saccades with practice and even while the fixation point remains visible (Fischer \& Breitmeyer, 1987). The implication here is that normal subjects may be able to voluntarily inhibit collicular pole fixation neurons even in the presence of a visual fixation stimulus. Under these circumstances, in which fixation cell activity is more under strategic control than under the exogenous influence of a fixation point, it may be expected that the FOE should be reduced. In Experiment 1, we examined whether normal adults can modulate the FOE strategically on the basis of oculomotor set when making saccades to peripheral visual targets (the VGR). In Experiment 2, we examined whether normal adults can modulate the FOE strategically on the basis of oculomotor set when making voluntary eye movements and, if so, whether the neural mech- anisms that control fixation for voluntary and reflexive eye movements might be independent of one another.

\section{EXPERIMENT 1}

In the first experiment, we looked at the effect of oculomotor bias or readiness to make a reflexive eye movement on the FOE. Each subject completed two test blocks. The frequency of activating the VGR was manipulated between blocks by varying the percentage of trials in which a target appeared at the periphery. The effect of offsetting the fixation point prior to appearance of the target on saccade latency was measured within each block and then compared between the two blocks. This experiment tested whether manipulating the frequency with which the VGR is activated affects the magnitude of the FOE.

\section{Method}

Subjects. Twenty undergraduates (mean age $=21, S D=4$ ) at the University of California, Berkeley, participated in this experiment in partial completion of a course requirement.

Stimuli and Procedure. Each subject completed 20 practice trials before each test block. There were two test blocks, each consisting of 200 trials. During one block, peripheral targets appeared in $20 \%$ of the trials, and in the other $80 \%$ no target appeared (catch trials). During the other block, peripheral targets appeared in $80 \%$ of the trials, and $20 \%$ were catch trials. The order of the two blocks was counterbalanced across subjects.

At the start of each trial, a fixation point, a $0.3^{\circ}$ white dot, appeared at the center of a black computer monitor. After $500 \mathrm{msec}$, an alerting tone sounded for $100 \mathrm{msec}$. The purpose of the tone was to control for the alerting effect associated with offsetting a fixation point prior to target onset (Reuter-Lorenz, Oonk, Barnes, \& Hughes, 1995). We were interested in measuring the oculomotor-specific effect of removing a fixation stimulus on saccade latency without the contribution of a general alerting effect. On half of the trials, the fixation point disappeared simultaneous with the onset of the tone (offset condition), and on the other half, the fixation point remained on the screen until the end of the trial (overlap condition). On targetpresent trials, a white asterisk subtending $1^{\circ}$ of visual angle appeared with equiprobability $8.8^{\circ}$ to the left or right of fixation $200 \mathrm{msec}$ after the tone terminated. Thus, on fixation offset trials, the temporal gap between fixation offset and target onset was $300 \mathrm{msec}$. The target and, on overlap trials, the fixation dot remained on the screen until either an eye movement was recorded or 1,000 msec lapsed. The screen was black for $2 \mathrm{sec}$ after each trial. Trial type (target or no target), target side (left or right), and fixation condition (offset or overlap) were all randomly intermixed.

The subjects sat $57 \mathrm{~cm}$ from a computer monitor and were instructed to fixate on the center of the screen until a target appeared in the periphery, at which time they should move their eyes to the target as quickly as possible. They were instructed to move their eyes back to the center of the screen after each eye movement and to maintain fixation until another target appeared in the periphery. Prior to starting each block, the subjects were informed which type of response would be most commonly correct (in this experiment, eye movements or fixating). On catch trials, the subjects were required to maintain fixation for $1 \mathrm{sec}$ after the onset of the alerting tone. Blinks and eye movements in the wrong direction, during catch trials, or with a latency of less than 70 msec triggered the computer to generate a loud beep for $500 \mathrm{msec}$, signaling that an error had been made. On target-present trials, if no response was recorded 
Table 1

Mean SRTs Saccadic Reaction Times (SRTs, in Milliseconds; With Standard Deviations) for Trials With Peripheral Saccade Targets (PSTs) in Experiment 1

\begin{tabular}{|c|c|c|c|c|c|}
\hline \multirow[b]{3}{*}{ Block } & \multicolumn{4}{|c|}{ SRTs } & \multirow[b]{3}{*}{ FOE } \\
\hline & \multicolumn{2}{|c|}{ Overlap } & \multicolumn{2}{|c|}{ Gap } & \\
\hline & $M$ & $S D$ & $M$ & $S D$ & \\
\hline $20 \%$ PST & 308 & 66 & 259 & 52 & 49 \\
\hline $80 \%$ PST & 239 & 44 & 211 & 38 & 28 \\
\hline
\end{tabular}

Note-Data are shown for each fixation condition in each block. The fixation offset effect (FOE) is shown as a difference score (SRTs in the overlap condition minus SRTs in the gap condition).

within $1 \mathrm{sec}$, the error beep sounded. The subjects were encouraged to make as few errors as possible.

Saccade latencies of the left eye were recorded with millisecond accuracy, using an Eye Trac 210 infrared scleral reflectance device (Applied Science Laboratories) that was interfaced with a computer through an analog device (Greg Laird Associates) that computed the velocity of the Eye Trac signal. During each trial, an eye movement value was recorded when the left eye reached the horizontal velocity threshold, which was approximately $60^{\circ}$ per second. Different values were outputted for leftward and rightward movements. Only the first value was accepted on each trial; hence, corrective eye movements were not recorded. The subjects viewed the stimuli on a NEC Multisync video graphics array monitor. The experimenter viewed both the stimuli and the position of the subject's eye on a different monitor and recalibrated when necessary.

\section{Results}

In both Experiments 1 and 2, practice trials were not analyzed. Also, test trials with correct saccadic reaction times (SRTs) lying outside three standard deviations above or below each subject's mean SRT in each condition were excluded from analysis $(1.1 \%$ of the trials in Experiment 1 and $2.0 \%$ of the trials in Experiment 2).

Saccade latencies. An analysis of variance (ANOVA) of the remaining correct saccade latencies showed no difference between left and right saccade targets and no interactions with block or fixation condition $(p>.1)$; thus, mean SRTs were collapsed across field of target. An ANOVA, with test block ( $20 \%$ reflexive saccades vs. $80 \%$ reflexive saccades) and fixation condition (overlap vs. gap) as factors, revealed that SRTs were faster in the block consisting of $80 \%$ peripheral targets than in the block consisting of only $20 \%$ peripheral targets $[F(1,19)=57.036$, $p<.001]$. There was also a main effect of fixation offset, so that SRTs were faster when the fixation point offset prior to target onset than when it remained on the screen until response $[F(1,19)=48.439, p<.001]$. The magnitude of the FOE interacted with the frequency of peripheral targets $[F(1,19)=10.246, p=.005]$, reflecting less benefit of removing the fixation stimulus on SRTs in the block consisting of $80 \%$ peripheral targets than in the block consisting of $20 \%$ peripheral targets. See Table 1 for a summary of the SRTs from Experiment 1.

Errors. Excluding trials during which the eye tracker was triggered by a blink, errors were recorded on $1.3 \%$ of the trials with a peripheral saccade target, including $0.4 \%$ anticipations (SRT $<70 \mathrm{msec}$ ), and on $2.4 \%$ of the catch trials, including $0.2 \%$ anticipations.

\section{Discussion}

The fixation stimulus affected SRTs less in the block with $80 \%$ peripheral targets than in the block with only $20 \%$ peripheral targets, as demonstrated by a smaller FOE. The change in the effectiveness of a fixation stimulus on saccade latencies may reflect changes in neural activity within the SCs, induced by changing the frequency with which peripheral targets appeared in a block of trials. In the SCs, the discharge rate of fixation neurons is higher when a fixation stimulus is present (Munoz \& Wurtz, 1992). High levels of fixation cell activity are associated with longer SRTs, since fixation cell activity inhibits collicular movement cell activity (Munoz \& Istvan, 1998; Munoz \& Wurtz, 1993b). Conversely, low levels of fixation cell activity are associated with shorter SRTs. When peripheral targets requiring rapid foveation are expected (as in the $80 \%$ peripheral targets block), it is advantageous to tonically decrease the activity of fixation cells so that eye movements to the targets can be carried out more quickly. Endogenous modulation of fixation cell activity may reduce the effect of external stimulation of these cells and, hence, the FOE. Strategic modulation of collicular fixation cell activity may be accomplished through corticocollicular projections, possibly originating in the frontal eye field (FEF) - an area involved in suppressing the VGR (Guitton, Buchtel, \& Douglas, 1985; Rafal, Machado, Ro, \& Ingle, 2000).

In 1992, Jüttner and Wolf investigated the effect of varying the likelihood of catch trials from $0 \%$ to $90 \%$ on the frequency of producing express saccades. In their experiment, the fixation stimulus disappeared $200 \mathrm{msec}$ before target onset on every trial, and the target always appeared to the left of fixation. Their results showed that the percentage of express saccades increased and mean saccade latency decreased with decreasing catch trials. Kingstone and Klein (1993b) also found more express saccades and shorter SRTs with $0 \%$ catch trials than with $20 \%$ catch trials; however, more important, they found that saccades that fell into the 80-120 msec express range, when target position was fixed, appeared to be anticipations rather than responses to the target.

With target position randomized, we found shorter saccade latencies in the block with fewer catch trials, yet a smaller FOE. A decrease in the size of the FOE can result from longer SRTs in the offset condition or from shorter SRTs in the overlap condition. Since SRTs decreased with decreasing catch trials, it seems unlikely that the decrease in the FOE in our $20 \%$ catch trials block resulted from longer SRTs on fixation offset trials. Rather, it is likely that the smaller FOE was largely an effect of shorter saccade latencies in the overlap condition. That is, it seems that the presence of a fixation stimulus slowed saccade initiation less in the $20 \%$ catch trials block than in the $80 \%$ catch trials block. 
There are several possible explanations for the modulation of the FOE observed in Experiment 1. As was proposed at the beginning of this discussion, one possibility is that it reflects a sensitivity to the expectation of making a reflexive eye movement. Another possibility is that it reflects an effect of general oculomotor preparationthat is, a sensitivity to the expectation of moving the eyes. These first two possible accounts are conflated in Experiment 1 , because the block in which fewer peripheral targets triggered the VGR also required fewer eye movements.

A third possibility is that the difference in the FOE between the two blocks resulted from a different distribution of visual attention. In the block with frequent peripheral targets, more attention may have been allocated to the periphery at the expense of fixation, and, therefore, events at fixation (i.e., offsets) may have had less influence on performance. This alternative seems unlikely, since Tam and Stelmach (1993) found equivalent FOEs (overlap vs. zero-gap conditions) when subjects endogenously attended either to the fixation stimulus or to a peripheral stimulus. Similarly, the size of the gap effect did not change when attention was exogenously drawn by a luminance change either to the fixation stimulus or to a peripheral location prior to fixation offset (Kingstone \& Klein, 1993a).

\section{EXPERIMENT 2}

Experiment 2 had three purposes: first, to determine which of the possible mechanisms is more likely to account for the modulation of the FOE observed in Experiment 1 ; second, to determine whether the fixation reflex (as measured by the FOE) also can be strategically controlled when individuals make voluntary eye movements; and third, if it can be so controlled, to determine whether strategic control over fixation for reflexive eye movements and that for voluntary eye movements share a common mechanism or are under independent control. In Experiment 2, the probability of a peripheral target was manipulated as in Experiment 1. However, an eye movement was made on every trial. Instead of catch trials, such as were used in Experiment 1, those trials without peripheral targets had nondirectional auditory tones as targets that instructed the execution of a voluntary eye movement. That is, the subjects were required to move their eyes in the direction arbitrarily assigned to the pitch of the tone (e.g., a high tone required a leftward saccade, and a low tone required a rightward saccade). Thus, although a saccade was made on every trial, the relative probability of a reflexive versus a voluntary saccade was manipulated between blocks. This enabled us to measure the FOE for both reflexive and voluntary saccades and to determine whether a strategic set to make one or the other type of saccade had different effects on the FOE for each type of eye movement.

Recent investigations of the FOE found that removal of a fixation stimulus also reduces the latency of voluntary saccades (Abrams, Oonk, \& Pratt, 1998; Forbes \& Klein, 1996). This is consistent with neurophysiological data in monkeys that showed that active fixation increases the minimum electrical current needed to evoke a saccade by stimulating the FEF (Goldberg, Bushnell, \& Bruce, 1986). When a higher activity threshold must be met in order for movement cells to generate a saccade, more time may be required, increasing saccade latency.

\section{Method}

Subjects. Twenty undergraduates (mean age $=20, S D=3$ ) from the same population as that in Experiment 1 were included in this experiment. Eight additional subjects completed this experiment; however, owing to the difficulty of this task, they did not meet our performance criterion of at least $65 \%$ correct for both reflexive and voluntary saccades in each block and so were excluded from all analyses.

Stimuli and Procedure. This experiment differed from Experiment 1 in the following ways: (1) On trials without a peripheral target, either a high- or a low-pitched tone sounded for $50 \mathrm{msec}$ from a single speaker in the computer case, and the subjects were required to make an eye movement in the direction indicated by the pitch of the tone; (2) the fixation point appeared for a temporal interval that varied randomly from 400 to $800 \mathrm{msec}$ (in $100-\mathrm{msec}$ steps) prior to target onset; (3) on fixation offset trials, the temporal gap between fixation offset and target onset was reduced to $0 \mathrm{msec}$; (4) there was no alerting tone; and (5) the subjects completed 40 practice trials and 240 test trials per block.

The subjects were asked to make an eye movement as quickly as they accurately could on each trial. The assignment of the two tones to rightward and leftward eye movements was counterbalanced between subjects, so that half of the subjects moved their eyes to the left side of the computer monitor in response to high tones and to the right side of the monitor in response to low tones, and the other half did the reverse. The tone-saccade mapping was also counterbalanced with the order of the two test blocks.

The gap procedure was abandoned in favor of a simultaneous (step) FOE manipulation for several reasons. Since auditory tones served as targets in this experiment, the use of auditory warning signals was impractical and would have been confusing to the subjects. Furthermore, other pilot work in our laboratory showed that the use of auditory tones may not completely control for the general alerting effect associated with removing the fixation stimulus prior to target onset (Reuter-Lorenz et al., 1995). Manual responses were also faster when the fixation stimulus offset than when it remained on the screen. In the gap condition, the subjects were alerted both auditorily (by the tone) and visually (by the offset of the fixation stimulus), whereas, in the overlap condition, the subjects were alerted only by the tone. Thus, to ensure that a visual alerting effect that was not oculomotor specific did not play a role in the modulation of the FOE, we eliminated the temporal gap between fixation offset and target onset on the fixation offset trials (Tam \& Stelmach, 1993). Finally, the recent report of Fendrich et al. (1999) suggests that the simultaneous procedure provides a more specific measure of the state of fixation neurons. Because the present study is concerned with strategic modulation of the oculomotor-specific FOE, not of the gap effect, we felt it would be useful to extend the observation of modulation shown in Experiment 1 by demonstrating that a general alerting effect was not a critical factor in the modulation.

\section{Results}

Saccade latencies. An ANOVA of the SRTs was computed with test block ( $80 \%$ reflexive saccades vs. $80 \%$ voluntary saccades), type of eye movement (reflexive vs. 
Table 2

Mean Saccadic Reaction Times (SRTs, in Milliseconds, With Standard Deviations) for Trials With Peripheral Saccade Targets (PSTs) on the Left and for Trials With Tone Saccade Targets on the Right in Experiment 2

\begin{tabular}{|c|c|c|c|c|c|c|c|c|c|c|}
\hline \multirow[b]{3}{*}{ Block } & \multicolumn{5}{|c|}{ Reflexive Saccade Trials } & \multicolumn{5}{|c|}{ Voluntary Saccade Trials } \\
\hline & \multicolumn{2}{|c|}{ Overlap } & \multicolumn{2}{|c|}{ Offset } & \multirow[b]{2}{*}{ FOE } & \multicolumn{2}{|c|}{ Overlap } & \multicolumn{2}{|c|}{ Offset } & \multirow[b]{2}{*}{ FOE } \\
\hline & $M$ & $S D$ & $M$ & $S D$ & & $M$ & $S D$ & $M$ & $S D$ & \\
\hline $20 \% \mathrm{PST} / 80 \%$ tone & 359 & 58 & 300 & 55 & 59 & 424 & 85 & 411 & 89 & 13 \\
\hline $80 \%$ PST $/ 20 \%$ tone & 277 & 48 & 251 & 39 & 26 & 557 & 132 & 518 & 123 & 39 \\
\hline
\end{tabular}

Note-The fixation offset effect (FOE) is shown as a difference score (SRTs in the overlap condition minus SRTs in the offset condition).

voluntary), and fixation condition (overlap vs. offset) as within-subjects variables. Saccade latencies were significantly shorter in the $80 \%$ reflexive saccade block than in the $80 \%$ voluntary saccade block $[F(1,19)=10.782, p=$ $.004]$, reflecting the fact that, overall, reflexive saccades had shorter latencies than did voluntary saccades $[F(1,19)=$ $75.150, p<.001]$. There was a main benefit of fixation offset on SRTs $[F(1,19)=43.891, p<.001]$. Each type of eye movement was faster in the block that required mostly eye movements of that type $[F(1,19)=169.911$, $p<.001]$. The type of eye movement also interacted with the fixation condition $[F(1,19)=5.610, p=.027]$, reflecting a more robust FOE for reflexive saccades $(42 \mathrm{msec})$ than for voluntary saccades $(25 \mathrm{msec})$. Of particular interest, there was a triple interaction between the test block, the type of eye movement, and the fixation condition $[F(1,19)=17.531, p<.001]$. The overall magnitude of the FOE did not differ between the two blocks $(F<1)$.

In order to assess the effect of the strategic set utilized during each test block on the FOE for each type of eye movement, separate ANOVAs were computed for peripheral visual target trials and tone target trials. An analysis of the trials with peripheral targets showed that, as in Experiment 1, there was an interaction between test block and fixation condition $[F(1,19)=11.979, p=.003]$, reflecting a smaller FOE in the $80 \%$ peripheral targets block ( $\mathrm{FOE}=26 \mathrm{msec}$ ) than in the $20 \%$ peripheral targets block (FOE $=59 \mathrm{msec})$. The FOE for reflexive saccades was revealed by $t$ tests to be reliable in both test blocks $(p<.001)$.

An analysis of the trials with tone targets showed that the FOE was smaller in the $80 \%$ tone block than in the $20 \%$ tone block $[F(1,19)=5.639, p=.027]$. The FOE for voluntary saccades was revealed by $t$ tests to be reliable in both test blocks $(p<.05)$. See Table 2 for a summary of the saccade latencies.

Errors. Error trials during which the eye tracker was triggered by a blink were excluded. Of the trials that required reflexive eye movements, a remaining $6.2 \%$ were counted as errors: No response was made on $3.2 \%, 0.3 \%$ were anticipations (SRT $<70 \mathrm{msec}$ ), and $2.7 \%$ were direction errors. Of the trials that required voluntary eye movements, a remaining $8.1 \%$ were counted as errors: no response was made on $1.6 \%, 0.1 \%$ were anticipations (SRT $<70 \mathrm{msec}$ ), and $6.4 \%$ were direction errors.

\section{Discussion}

Experiment 2 confirmed that the FOE for reflexive saccades was modulated by strategic set, as in Experiment 1 , and that strategic set also modulated the FOE for voluntary saccades. The modulation of the FOE for each type of eye movement was specific to that type of eye movement. In the block with frequent reflexive eye movements, the FOE for reflexive eye movements was reduced, but the FOE for voluntary eye movements was not reduced. The reverse was true in the block with frequent voluntary eye movements. The dissociation in the modulation of the FOE for different types of saccades under the same strategic set suggests that the FOE for reflexive saccades and that for voluntary saccades may reflect fixationdependent neural activity changes in two different parts of the brain: possibly, the SC for the reflexive and the FEF for the voluntary.

The ability to make voluntary eye movements evolved more recently than the VGR and requires cortical control. Converging evidence in humans has demonstrated that the FEF controls voluntary saccades (Henik, Rafal, \& Rhodes, 1994; Paus, 1996; Ro, Cheifet, Ingle, Shoup, \& Rafal, 1999; Ro, Henik, Machado, \& Rafal, 1997). Singleunit recording and microstimulation in primates suggests that the FEF may control saccades in a manner similar to that by which the SC controls reflexive eye movements. Like the SC, the FEF contains movement cells that are capable of moving the eyes and fixation cells whose activity can hold the eyes (Burman \& Bruce, 1997; Hanes, Patterson, \& Schall, 1998). Orchestration of the activity of these two types of cells may determine voluntary eye movements. In addition, FEF fixation cells may play a critical role in suppressing contraversive reflexive eye movements generated by the SC, since unilateral damage to the FEF impairs suppression of the VGR toward stimuli suddenly appearing in the contralesional visual field (Rafal et al., 2000).

Overall, the FOE for voluntary eye movements in Experiment 2 was smaller than the FOE for reflexive eye movements. Abrams et al. (1998) also reported a smaller FOE for voluntary than for reflexive eye movements; 
however, Forbes and Klein (1996) reported equivalent FOEs. The present results indicate that the size of the FOE for both reflexive and voluntary saccades depends on the strategic set, which is determined by the circumstances at hand. Thus, it may not be relevant to ask whether the FOE is larger or smaller for one type of saccade than for the other in absolute terms, and it may not be surprising that the magnitude of the FOE for the two types of eye movement varies across different studies.

Two possible alternative explanations of the modulation of the FOE in Experiment 1 were ruled out by the results of the present experiment. First, modulation of the FOE is not attributable to a general readiness to move the eyes; an eye movement was required on every trial in the present experiment. Second, the smaller FOE for reflexive saccades when peripheral visual targets were frequent cannot be explained by less attention focused on the fixation stimulus. As was mentioned in the previous discussion, the subjects may have allocated less attention to the fixation stimulus in the $80 \%$ peripheral targets block in favor of attending to the two peripheral saccade target locations, since the occurrence of peripheral targets was frequent; in the $20 \%$ peripheral targets block, they may have focused their attention more on fixation, since the correct response on most trials was to maintain fixation. However, the modulation of the FOE cannot be explained by a change in the amount of attention directed at fixation since, in the present experiment, in the block with $80 \%$ peripheral visual targets, the FOE was smaller for reflexive saccades but larger for voluntary saccades, and the reverse was true in the block with $20 \%$ peripheral targets.

\section{GENERAL DISCUSSION}

The results of the two experiments presented here show that individuals are able to modulate the effect of a fixation point on SRTs on the basis of strategic set. We demonstrated that increasing the frequency of peripheral targets reduced the effect of a fixation stimulus in increasing the latencies of reflexive eye movements. This modulation suggests that fixation cells in the rostral poles of the SCs are under cortical control (possibly originating in the FEFs) and that their activity can be strategically regulated independent of the presence or absence of a visual signal at fixation. When peripheral targets requiring rapid foveation appear frequently, the frontal cortex may send a strategic inhibitory command to collicular fixation cells in an effort to minimize reflexive saccade latencies. This endogenous command to tonically decrease fixation cell activity may attenuate the excitatory effect of an external visual signal at fixation on fixation cells. Thus, it seems that strategic set can bring fixation cell activity under voluntary control and make it more autonomous with respect to external stimulation.

As with reflexive eye movements, varying the frequency of voluntary eye movements modified the control of a visual stimulus at fixation on the latencies of vol- untary saccades. The discrepancy in the size of the FOE for reflexive and voluntary eye movements under the same strategic set indicates that the reliance of reflexive saccade latencies and the reliance of voluntary saccade latencies on the presence of a fixation stimulus are independent of each other. This independence suggests that strategic control of fixation for reflexive saccades and voluntary saccades are mediated by different cell populations. We propose that the FOE for voluntary saccades may reflect a change in the activity of fixation cells in the FEF, whereas the FOE for reflexive saccades reflects the activity of fixation cells in the SC. Like SC fixation cells, FEF fixation cells may be under cortical control that changes with strategic set, accounting for the change in the dependence of voluntary saccade latencies on the presence of a fixation stimulus between the two test blocks.

Several other saccade paradigms also involve strategic sets that reduce the effect of a fixation stimulus on saccade latencies. The FOE for antisaccade tasks, which require eye movements away from peripheral targets, has been shown to be smaller than that for prosaccade tasks, which require eye movements toward peripheral targets (Forbes \& Klein, 1996; Reuter-Lorenz et al., 1991; ReuterLorenz et al., 1995). In antisaccade tasks, during which reflexive saccades must be inhibited, subjects may adopt a strategic set that results in a tonically higher rate of fixation cell activity (Everling, Dorris, Klein, \& Munoz, 1998), which may continue even when the fixation point offsets. The effect of fixation offset on SRTs is also reduced in prosaccade tasks when target location is cued, enabling preparation of an eye movement, prior to target onset (Rafal et al., 2000). This finding suggests that fixation cell activity can be phasically decreased by voluntary preparation even when a fixation stimulus is present. Similarly, Taylor, Kingstone, and Klein (1998) reported a reduction in the FOE when the timing of target onset was made predictable and attributed this disinhibition of the saccadic system to top-down processes.

Neurophysiological data recorded in monkeys performing a gap task also suggest that the FOE may be strategically modulated on the basis of how often the VGR is expected to be activated by peripheral signals (Dias \& Bruce, 1994). During a gap task, where stimuli requiring a prosaccade response appeared in the periphery, neurons in the FEF increased their firing rate when the fixation stimulus disappeared. However, during a control gap task in which stimuli never appeared in the periphery and a reward was only given if the monkey maintained fixation, the FEF discharge associated with fixation offset diminished with increasing trials.

In summary, the effect of a visual signal at fixation on reflexive and voluntary saccade latencies depends on strategic set. Moreover, the extent to which a visual signal at fixation increases reflexive saccade latencies is independent of the extent to which a fixation stimulus increases voluntary saccade latencies. This independence suggests that the FOEs for reflexive and voluntary saccades depend on changes in the activity of different cell 
populations. Neurophysiological studies of monkeys suggest that the FOE for reflexive saccades may depend on a change in fixation cell activity in the SC (Munoz \& Wurtz, 1992). We propose that the FOE for voluntary saccades may depend on a change in fixation cell activity in the FEF.

\section{REFERENCES}

Abrams, R. A., Oonk, H. M., \& Pratr, J. (1998). Fixation point offsets facilitate endogenous saccades. Perception \& Psychophysics, 60, 201-208.

Burman, D. D., \& BruCE, C. J. (1997). Suppression of task-related saccades by electrical stimulation in the primate's frontal eye field. Journal of Neurophysiology, 77, 2252-2267.

BÜTTNER-ENNEVER, J. A., \& HoRn, A. K. E. (1997). Anatomical substrates of oculomotor control. Current Opinion in Neurobiology, 7, 872-879.

DiAs, E. C., \& BRUCE, C. J. (1994). Physiological correlate of fixation disengagement in the primate's frontal eye field. Journal of Neurophysiology, 72, 2532-2537.

Dorris, M. C., \& Munoz, D. P. (1995). A neural correlate for the gap effect on saccadic reaction times in monkey. Journal of Neurophysiology, 73, 2558-2562.

Everling, S., Dorris, M. C., Klein, R. M., \& Munoz, D. P. (1998). Superior colliculus neuronal activity in monkeys during pro- and antisaccade tasks. Paper presented at the 28th annual meeting of the Society for Neuroscience, Los Angeles.

Fendrich, R., Demirel, S., \& Danziger, S. (1999). The oculomotor gap effect without a foveal fixation point. Vision Research, 39, 833841.

FisChER, B., \& BREITMEYER, B. (1987). Mechanisms of visual attention revealed by saccadic eye movements. Neuropsychologia, 25, 73-84.

FisCHER, B., \& RAMSPERGER, E. (1984). Human express saccades: Extremely short reaction times of goal directed eye movements. Experimental Brain Research, 57, 191-195.

FORBES, K., \& KLEIN, R. M. (1996). The magnitude of the fixation offset effect with endogenously and exogenously controlled saccades. Journal of Cognitive Neuroscience, 8, 344-352.

GoldBerg, M. E., Bushnell, M. C., \& Bruce, C. J. (1986). The effect of attentive fixation on eye movements evoked by electrical stimulation of the frontal eye fields. Experimental Brain Research, 61, 579-584.

GuitTon, D., BuChTEl, H. A., \& Douglas, R. M. (1985). Frontal lobe lesions in man cause difficulties in suppressing reflexive glances and in generating goal directed saccades. Experimental Brain Research, 58, $455-472$.

Hanes, D. P., Patterson, W. F., II, \& Schall, J. D. (1998). Role of frontal eye fields in countermanding saccades: Visual, movement, and fixation activity. Journal of Neurophysiology, 79, 817-834.

HeniK, A., Rafal, R., \& RHODEs, D. (1994). Endogenously generated and visually guided saccades after lesions of the human frontal eye fields. Journal of Cognitive Neuroscience, 6, 400-411.

Hood, B. M., ATkInson, J., \& Braddick, O. J. (1998). Selection-foraction and the development of visual selective attention. In J. E. Richards (Ed.), Cognitive neuroscience of attention: A developmental perspective (pp. 219-250). Hillsdale, NJ: Erlbaum.

JOHNSON, M. H. (1990). Cortical maturation and the development of visual attention in early infancy. Journal of Cognitive Neuroscience, 2 , 81-95.

Johnson, M. H., \& GILMORE, R. O. (1998). Toward a computational model of the development of saccade planning. In J. E. Richards (Ed.), Cognitive neuroscience of attention: A developmental perspective (pp. 103-130). Hillsdale, NJ: Erlbaum.

JÜTTNER, M., \& WOLF, W. (1992). Occurrence of human express saccades depends on stimulus uncertainty and stimulus sequence. $E x$ perimental Brain Research, 89, 678-681.

KINGSTONE, A., \& KLEIN, R. M. (1993a). Visual offsets facilitate saccadic latency: Does predisengagement of visuospatial attention mediate this gap effect? Journal of Experimental Psychology: Human Perception \& Performance, 19, 1251-1265.
Kingstone, A., \& KLEIN, R. M. (1993b). What are human express saccades? Perception \& Psychophysics, 54, 260-273.

KLEIN, R. M., \& Kingstone, A. F. (1993). Why do visual offsets reduce saccadic latencies. Behavioral \& Brain Sciences, 16, 583-584.

Munoz, D. P., \& IstVan, P. J. (1998). Lateral inhibitory interactions in the intermediate layers of the monkey superior colliculus. Journal of Neurophysiology, 79, 1193-1209.

MUNOz, D. P., \& WURTZ, R. H. (1992). Role of the rostral superior colliculus in active visual fixation and execution of express saccades. Journal of Neurophysiology, 67, 1000-1002.

Munoz, D. P., \& WURTZ, R. H. (1993a). Fixation cells in monkey superior colliculus: I. Characteristics of cell discharge. Journal of Neurophysiology, 70, 559-575.

Munoz, D. P., \& WuRTZ, R. H. (1993b, November). Interactions between fixation and saccade neurons in primate superior colliculus. Paper presented at the 23rd annual meeting of the Society for Neuroscience, Washington, DC.

PARÉ, M., \& GuITTON, D. (1994). The fixation area of the cat superior colliculus: Effects of electrical stimulation and direct connection with brainstem omnipause neurons. Experimental Brain Research, 101, 109-122.

PAus, T. (1996). Location and function of the human frontal eye-field: A selective review. Neuropsychologia, 34, 475-483.

Rafal, R., Machado, L., Ro, T., \& INGLE, H. (2000). Looking forward to looking: Saccade preparation and control of the visual grasp reflex. In S. Monsell \& J. Driver (Eds.), Attention and performance XVIII: Control of cognitive processes (pp. 155-174). Cambridge, MA: MIT Press.

Reuter-Lorenz, P. A., Hughes, H. C., \& Fendrich, R. (1991). The reduction of saccadic latency by prior offset of the fixation point: An analysis of the gap effect. Perception \& Psychophysics, 49, 167-175.

Reuter-Lorenz, P. A., OONK, H. M., Barnes, L. L., \& Hughes, H. C. (1995). Effects of warning signals and fixation point offsets on the latencies of pro-versus antisaccades: Implications for an interpretation of the gap effect. Experimental Brain Research, 103, 287-293.

Ro, T., Cheifet, S., Ingle, H., Shoup, R., \& Rafal, R. (1999). Localization of the human frontal eye fields and motor hand area with transcranial magnetic stimulation and magnetic resonance imaging. Neuropsychologia, 37, 225-231.

Ro, T., Henik, A., Machado, L., \& Rafal, R. D. (1997). Transcranial magnetic stimulation of the prefrontal cortex delays contralateral endogenous saccades. Journal of Cognitive Neuroscience, 9, 433-440.

Ross, S. M., \& Ross, L. E. (1981). Saccade latency and warning signals: Effects of auditory and visual stimulus onset and offset. Perception \& Psychophysics, 29, 429-437.

SASLOW, M. G. (1967). Effects of components of displacement-step stimuli upon latency for saccadic eye movements. Journal of the Optical Society of America, 57, 1024-1029.

TAM, W. J., \& Stelmach, L. B. (1993). Viewing behavior: Ocular and attentional disengagement. Perception \& Psychophysics, 54, 211 222.

TAYlOR, T. L., Kingstone, A., \& Klein, R. M. (1998). The disappearance of foveal and nonfoveal stimuli: Decomposing the gap effect. Canadian Journal of Experimental Psychology, 52, 192-199.

WurTz, R. H., \& MuNOz, D. P. (1995). Role of monkey superior colliculus in control of saccades and fixation. In M. S. Gazzaniga (Ed.), The cognitive neurosciences (pp. 533-548). Cambridge, MA: MIT Press, Bradford Books.

\section{NOTE}

1. There are several different types of eye-movement-related cells in the deeper layers of the SC. For example, buildup cells can be found caudal to the fixation cells, and burst cells can be found in a layer superficial to the fixation cells.

(Manuscript received May 7, 1998; revision accepted for publication October 6, 1999.) 